\title{
Effect of Temperature on Devulcanization of Waste Sidewall Rubber by Supercritical Ethanol
}

\author{
Xiang Li, ${ }^{a}$ Xiao-Qing Deng ${ }^{*, b}$ and Chao Dong ${ }^{c}$ \\ ${ }^{a}$ School of Mechanical Engineering, Yangtze University, 434023 Jingzhou, P. R. China \\ ${ }^{b}$ College of Chemistry and Environmental Engineering, Yangtze University, \\ 434023 Jingzhou, P. R. China \\ ${ }^{c}$ Faculty of Chemical, Environmental and Biological Science and Technology, \\ Dalian University of Technology, 116024 Dalian, P. R. China
}

\begin{abstract}
The influence of temperature on devulcanization of waste sidewall rubber via supercritical ethanol was investigated. The effect of parameters on devulcanizing process was analyzed by a full factorial experimental design. The devulcanized products at different temperatures were characterized by gel permeation chromatography (GPC), differential scanning calorimetry (DSC), thermogravimetry and derivative thermogravimetry (TG-DTG) and Fourier transform infrared (FTIR) spectrophotometry. In addition, the kinetics analysis for the devulcanizing process via supercritical ethanol was established. It was found that the reaction temperature was the only significant parameter. When the temperature rose above $240{ }^{\circ} \mathrm{C}$, the reaction rate increased dramatically. A special phenomenon was observed that the devulcanizing reaction rate of natural rubber (NR) was faster than that of butadiene rubber (BR) in supercritical ethanol. Moreover, when the reaction temperature reached $270{ }^{\circ} \mathrm{C}$, the use of the devulcanizing reagent did not significantly affect the sol fraction. The analysis of experimental results indicated that supercritical ethanol beyond the critical temperature could strongly promote the devulcanizing reaction, maintaining polymer structure of products as the most intact state.
\end{abstract}

Keywords: supercritical ethanol, waste sidewall rubber, temperature, kinetics

\section{Introduction}

The recycling of waste tire rubber has attracted intensive attention due to the great efforts related to the prevention of environmental pollution and conservation of petroleum resource. However, the tire rubber is difficult to be recycled due to the presence of its stable three-dimensional crosslinks. Currently, devulcanization is widely applied in the field of the waste rubber recycling. Through the devulcanizing process, the three-dimensional crosslinked network is broken and the devulcanized waste tire has the ability to reformulate and recycle into usable products. ${ }^{1}$ Various types of devulcanizing routes have been studied so far in order to improve the quality of the recycled products, such as chemical method, mechanical process, microwave technique, ultrasonic method and microbial method. ${ }^{2-13}$ However, some processes have weaknesses such as environmental pollution and low efficiency.

*e-mail: dxq_j18@163.com
Supercritical fluid devulcanizing technology is increasingly regarded as an available method for the devulcanization due to its environmental advantage and high efficiency. Crosslinked polymer could be recycled by employing supercritical alcohol. The crosslinked polyethylene has been selectively decomposed by supercritical alcohol and the recycled product could undergo the crosslinking reaction. ${ }^{14}$ Furthermore, supercritical alcohol is a kind of solvent which has high chemical activity and could replace the catalyst in some cases. Such as in the degradation process of the alkali lignin, the use of a catalyst did not significantly affect the yields in both solvent systems with alcohol and water. ${ }^{15}$ In the field of tire rubber recovery, Kershaw ${ }^{16}$ tried to degrade tire rubber to obtain the low molecular weight oil by supercritical ethanol, but most products maintained the macromolecular structure after reaction, which was very close to the devulcanization process. In our previous work, ${ }^{17}$ the sidewall rubber was also devulcanized effectively in supercritical ethanol, and the production exhibited the high sol fraction (SF) and lower crosslinking density (CD) of gel. 
As is well known, temperature plays a very important role in the recycling of the crosslinked polymer by supercritical fluid. Increased reaction temperature could increase the amount of radicals and enhance the mobility of monomer, which results in a higher degradation rate of crosslinked network. In $\mathrm{ScCO}_{2}$ devulcanizing process, the temperature was evaluated as the most significant factor by mathematical statistical method. ${ }^{18,19}$ Meanwhile, the temperature was also the deciding factor for decrosslinking of polymer in supercritical alcohol. ${ }^{20}$ Under some conditions, increased temperature not only accelerates the degradation of crosslinked network in dynamics, but also changes the activity of the supercritical fluids, which will further promote the reaction. Su et al. ${ }^{21}$ achieved the degradation of polyethylene by using supercritical water, and demonstrated that the temperature has significant impact on both the degradation of crosslinked network and the activity of supercritical fluid. However, to the best of our knowledge, few studies about the influence of temperature on the supercritical ethanol devulcanizing process are reported.

In this work, we investigated the role of temperature on devulcanization of the sidewall rubber in supercritical ethanol. A full factorial experimental design was used to analyze the devulcanizing process. The chemical structures and thermal properties of the products were characterized. The reaction rate constant of devulcanizing process was obtained. In addition, a possible reaction path by the effect of temperature was discussed.

\section{Experimental}

\section{Materials}

The experimental feedstock was sidewall rubber obtained from a passenger car tire (Dunlop tires, model 215/60R16 95H, China). The rough rubber pieces were extracted in the azeotropic acetone/chloroform (3/7 v/v) mixture for $12 \mathrm{~h}$ in a Soxhlet extractor to remove the low molecular weight compounds. The extracted pieces were dried to constant weight in vacuum at $50^{\circ} \mathrm{C}$, and then trimmed into strips $\left(20 \times 3 \times 3 \mathrm{~mm}^{3}\right)$ as the experimental samples. The experimental samples will be referred to as vulcanizate later. The devulcanizing reagent, diphenyl disulfide (DPDS), was purchased from Energy Chemical, China. Ethanol, acetone, chloroform and toluene were supplied by Sinopharm Chemical Reagent Beijing Co., Ltd, China.

\section{Characterization}

Devulcanized product was analyzed by an EQUINOX55 Fourier transform infrared (FTIR) spectrophotometer
(Germany), and the spectrum of sol was taken in the range of 4000 to $400 \mathrm{~cm}^{-1}$ with potassium bromide crystals. Gel permeation chromatography (GPC) was performed at $30^{\circ} \mathrm{C}$ with a TDA302 chromatograph (USA) to detect the molar mass and molar mass distributions (polydispersity index (PDI)) of sols. The glass transition temperature of vulcanizate and gel was characterized in the range of -100 to $40{ }^{\circ} \mathrm{C}$, with a heating rate of $10^{\circ} \mathrm{C} \mathrm{min}^{-1}$ in liquid nitrogen by a Q2000 differential scanning calorimeter (DSC) (USA). Thermogravimetry (TG) and derivative thermogravimetry (DTG) were performed in a nitrogen atmosphere with a SETSYS 16/18 thermogravimetric analyzer (France), at a heating rate of $10{ }^{\circ} \mathrm{C} \mathrm{min}^{-1}$ in the range of 0 to $650{ }^{\circ} \mathrm{C}$.

\section{Experimental process}

Devulcanizing reaction was performed in a stainless steel autoclave $(100 \mathrm{~mL}$, design pressure of $20 \mathrm{MPa}$ and design temperature of $350{ }^{\circ} \mathrm{C}$ ). The reaction process is shown in Figure 1. A piece of stainless steel net, which the vulcanizate was placed on, was installed in the middle of the autoclave, and the ethanol was placed on the bottom of the autoclave. Nitrogen was fed into the autoclave, and then discharged for removing the air. This process was repeated five times before starting the reaction. The autoclave was filled with a certain volume of ethanol at ambient temperature, and then followed by heating to the target temperature. The final pressure was referred to as the target pressure, which was only controlled by the volume of ethanol and the target temperature. The heat was provided by an electric heating furnace. The average heating rate was $5{ }^{\circ} \mathrm{C} \mathrm{min}^{-1}$. When the reaction was completed, the autoclave was taken out of the furnace and cooled by a blower to ambient temperature.

The devulcanized product was dried to constant weight $\left(\mathrm{W}_{\mathrm{d}}\right)$ in vacuum at the ambient temperature. If the devulcanizing reagent were added during the (a)

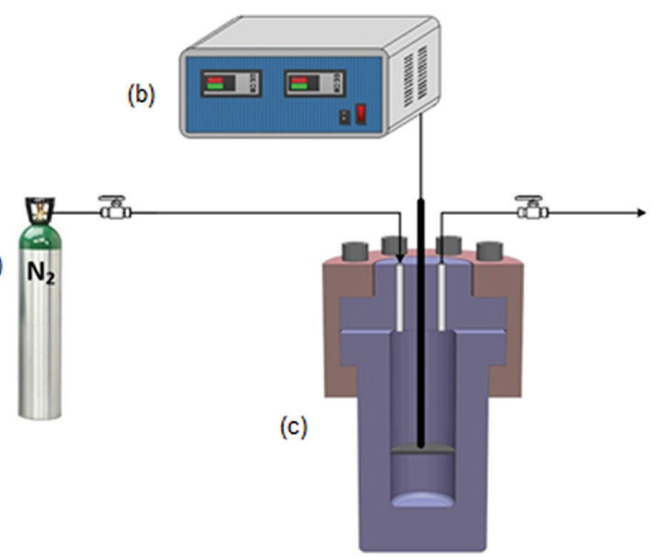

Figure 1. Scheme of the experimental process for devulcanization. (a) Cylinders; (b) control cabinet; and (c) autoclave. 
devulcanization process, the dried product would be extracted by acetone for $48 \mathrm{~h}$ to remove the unreacted devulcanizing reagent. Then the insoluble fraction was dried again to $\mathrm{W}_{\mathrm{d}}$ at the same conditions. Subsequently, the dried product was extracted by chloroform with a Soxhlet extractor for $24 \mathrm{~h}$ to obtain the soluble (sol) and insoluble (gel) products. The products were all dried to constant weight in vacuum at the ambient temperature. The sol was comprised of the liner rubber molecules, which were recyclable, and the gel still maintained the crosslinked structure. In general, the higher SF and lower CD of gel reflect a more efficient devulcanized process. The weights of dried sol and gel were $\mathrm{W}_{\mathrm{s}}$ and $\mathrm{W}_{\mathrm{g}}$, respectively. The SF was calculated as

Sol fraction $=\frac{\mathrm{W}_{\mathrm{s}}}{\mathrm{W}_{\mathrm{d}} \times \mathrm{W}} \times 100 \%$

where $\mathrm{w}$ is the weight percentage of rubber ingredient in the sidewall rubber.

The $\mathrm{CD}$ of vulcanizate and gel swollen in toluene was calculated by the Flory-Rehner equation: ${ }^{22}$

$\mathrm{v}=\frac{-\left[\ln \left(1-\mathrm{v}_{\mathrm{r}}\right)+\mathrm{v}_{\mathrm{r}}+\mu \mathrm{v}_{\mathrm{r}}^{2}\right]}{\mathrm{V}_{0}\left[\mathrm{v}_{\mathrm{r}}^{1 / 3}-\left(\mathrm{v}_{\mathrm{r}} / 2\right)\right]}$

where $\mathrm{v}$ is the $\mathrm{CD} ; \mathrm{v}_{\mathrm{r}}$ is the volume fraction of the rubber crosslinked network in the swollen gel; $\mathrm{V}_{0}$ is the molar volume of the swelling solvent; and $\mu$ is the rubber-solvent interaction parameter, $\mu=0.39 .^{23}$

If the gel contained carbon black, $\mathrm{v}_{\mathrm{r}}$ was corrected by equation $3:{ }^{24}$

$\frac{\mathrm{v}_{\mathrm{r} 0}}{\mathrm{v}_{\mathrm{r}}}=0.56^{-\mathrm{z}}+0.44$

where $\mathrm{V}_{\mathrm{r} 0}$ is the corrected volume fraction of the rubber

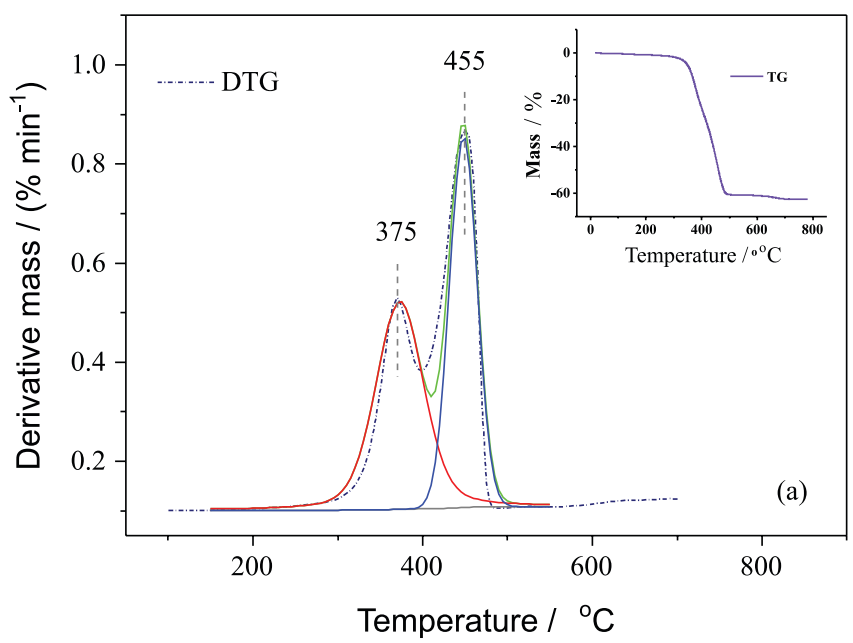

Figure 2. Thermal analysis of the vulcanizate. (a) TG-DTG and (b) DSC. crosslinked network in the swollen gel and $\mathrm{z}$ is the mass concentration of carbon black.

\section{Results}

Vulcanizate ingredients analysis

The conventional rubber compositions of tires are natural rubber (NR), styrene butadiene rubber (SBR), butadiene rubber (BR) and isobutylene isoprene rubber (IIR). ${ }^{25-28}$ In our previous experiment, ${ }^{17}$ we found that IIR was easy to be devulcanized, and even degraded. Therefore, IIR layer was removed before devulcanization experiment.

The ingredient of sidewall rubber was determined by TG-DTG, shown in Figure 2a. A mass loss of about $60 \%$ was observed in TG from 300 to $500{ }^{\circ} \mathrm{C}$ due to the degradation of the rubber ingredient. The peaks of DTG around 375 and $455{ }^{\circ} \mathrm{C}$ represented the maximum mass loss rate of NR and synthetic rubber (SBR or BR), respectively. After fitting analysis, the proportions of NR and synthetic rubber were 53.6 and $46.4 \%$, respectively, in rubber composition. To further analyze the types of synthetic rubber, the sol was extracted from completely devulcanized sidewall rubber as sample for DSC and the analysis result is shown in Figure 2b. It can be observed that two glass transitions occurred at -68 and $-101{ }^{\circ} \mathrm{C}$, and the latter corresponded to that of BR. Therefore, the synthetic rubber was $B R$ in the sidewall rubber.

\section{Full factorial experimental design}

A full factorial experimental design was used to analyze the influence of the parameters on devulcanizing process. The objective functions were SF and CD of gel. The levels of each factor were chosen by our previous study and are

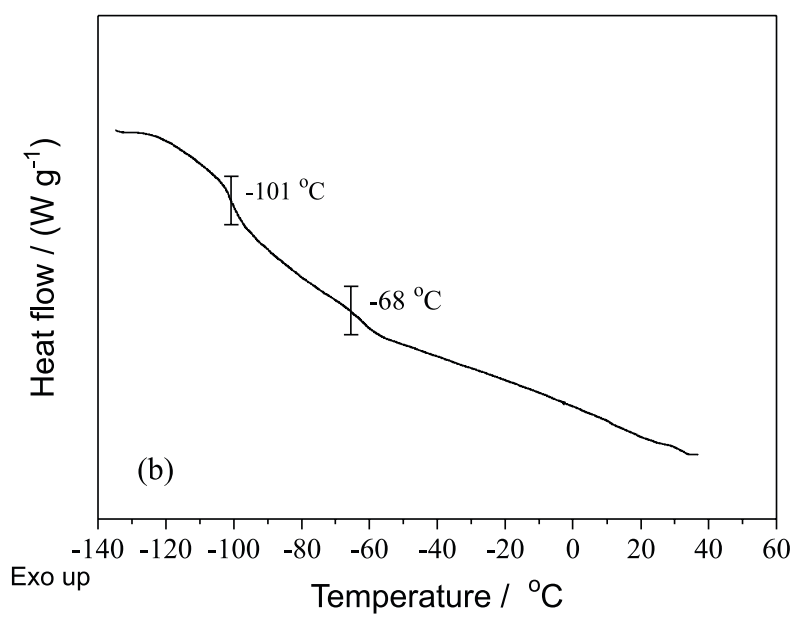


shown in Table $1 .{ }^{17}$ It is worth noting that the levels of pressure were all greater than the critical value of ethanol.

Table 1. Factors and levels of experimental design

\begin{tabular}{lccc}
\hline Factor code & $\begin{array}{c}\text { Minimum } \\
\text { level }-1\end{array}$ & $\begin{array}{c}\text { Central } \\
\text { level } 0\end{array}$ & $\begin{array}{c}\text { Maximum } \\
\text { level }+1\end{array}$ \\
\hline Temperature $/{ }^{\circ} \mathrm{C}$ & 200 & 230 & 260 \\
Pressure / MPa & 8 & 10 & 12 \\
time / min & 30 & 75 & 120 \\
\hline
\end{tabular}

The experimental design and its corresponding results are shown in Table 2. Three central point experiments were used to verify the accuracy, reproducibility and experimental error of regression model. The results of variant analyses are shown in Table 3. The influence of factors interaction on devulcanization was neglected because the main effect model agreed well with the experimental results. The probability value ( $p$-value) of temperature was lower than 0.05 for $\mathrm{SF}$ and $\mathrm{CD}$, and therefore temperature was proven to be the only significant factor. Pressure and time had no appreciable effect on devulcanization. The normal probability plots of the residuals for responses are shown in Figure 3; no abnormal experimental points were observed.

For further analysis, the influence of temperature on devulcanization was studied (Figure 4). It could be observed that, in a low temperature region (from 210 to $240{ }^{\circ} \mathrm{C}$ ), the SF increased gradually from 7 to $13 \%$ (Figure $4 a$ ). As the reaction temperature changed from 240 to $270{ }^{\circ} \mathrm{C}$, the SF rose dramatically from 13 to $55 \%$, however, it turned to depend weakly on the temperature around $56 \%$ in a higher temperature region (above $270{ }^{\circ} \mathrm{C}$ ). There was an obvious difference in the increase rate of SF below and above $240{ }^{\circ} \mathrm{C}$ which was around the critical temperature of ethanol. This result showed that the devulcanizing process was accelerated when the ethanol entered into the supercritical state. The CDs at different temperatures are also shown in Figure 4b, and it can be seen that the CDs of devulcanized samples are all less than that of vulcanizate.

Table 2. Full factorial design and experimental responses (SF and CD)

\begin{tabular}{|c|c|c|c|c|c|}
\hline Experiment & Temperature $/{ }^{\circ} \mathrm{C}$ & Pressure / MPa & time / min & $\mathrm{SF}^{\mathrm{a}} / \%$ & $\mathrm{CD}^{\mathrm{b}} /\left(\mathrm{mmol} \mathrm{cm}^{-3}\right)$ \\
\hline E1 & 260 & 8 & 120 & 36.96 & 0.133 \\
\hline E2 & 260 & 8 & 30 & 28.23 & 0.117 \\
\hline E3 & 200 & 8 & 30 & 3.53 & 0.141 \\
\hline $\mathrm{E} 4$ & 230 & 10 & 75 & 16.18 & 0.127 \\
\hline E5 & 260 & 12 & 30 & 33.00 & 0.069 \\
\hline E6 & 260 & 12 & 120 & 44.21 & 0.074 \\
\hline E7 & 230 & 10 & 75 & 18.17 & 0.132 \\
\hline E8 & 200 & 8 & 120 & 3.78 & 0.150 \\
\hline Center 1 & 200 & 12 & 120 & 4.22 & 0.141 \\
\hline Center 2 & 200 & 12 & 30 & 3.75 & 0.121 \\
\hline Center 3 & 230 & 10 & 75 & 15.38 & 0.122 \\
\hline
\end{tabular}

a Sol fraction; ${ }^{\mathrm{b}}$ crosslinking density of gel. E: experiment.

Table 3. Analysis and statistical factors of regression model of SF and CD

\begin{tabular}{|c|c|c|c|c|c|c|c|}
\hline \multirow{2}{*}{ Variable } & \multirow{2}{*}{$\mathrm{DF}^{\mathrm{a}}$} & \multicolumn{3}{|c|}{ SF } & \multicolumn{3}{|c|}{$\mathrm{CD}$} \\
\hline & & $\mathrm{SS}^{\mathrm{b}}$ & $F^{\mathrm{c}}$ & $p$-Value ${ }^{\mathrm{d}}$ & $\mathrm{SS}^{\mathrm{b}}$ & $F^{\mathrm{c}}$ & $p$-Value ${ }^{\mathrm{d}}$ \\
\hline Model & 3 & 2090.05 & 55.09 & $<0.0001$ & 0.00591 & 13.38 & 0.0028 \\
\hline $\mathrm{T}$ & 1 & 2017.36 & 159.52 & $<0.0001$ & 0.00324 & 21.99 & 0.0022 \\
\hline$P$ & 1 & 19.84 & 1.57 & 0.2506 & 0.00235 & 15.93 & 0.0052 \\
\hline $\mathrm{t}$ & 1 & 52.84 & 4.18 & 0.0802 & 0.00328 & 2.23 & 0.1794 \\
\hline Residual & 7 & 88.52 & & & 0.00103 & & \\
\hline Lack of fit & 5 & 84.37 & 8.12 & 0.1132 & 0.000986 & 8.86 & 0.1045 \\
\hline Pure error & 2 & 4.16 & & & 0.0000445 & & \\
\hline Corrected total & 10 & 2178.57 & & & 0.00694 & & \\
\hline
\end{tabular}

${ }^{\mathrm{a}}$ Degrees of freedom; ${ }^{\mathrm{b}} \mathrm{sum}$ of square deviations from the overall mean; ${ }^{\mathrm{c}} F$ distribution ratio; ${ }^{\mathrm{d}}$ probability value. SF: sol fraction; CD: crosslinking density of gel; T: temperature; P: pressure; t: time. 


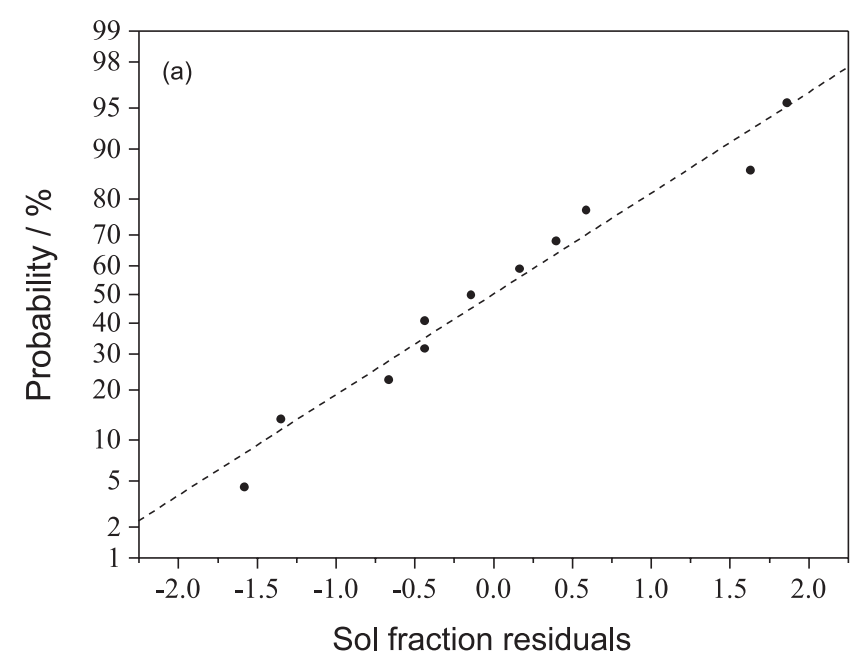

Figure 3. Normal probability plot of residuals for (a) SF and (b) CD of gel.

This implied that the supercritical ethanol permeated into the inside of vulcanizate and broke the crosslinked network. In supercritical ethanol, the $\mathrm{CD}$ of devulcanized samples decreased as the temperature goes up, and then, showed a slight increase when the temperature reached $270{ }^{\circ} \mathrm{C}$. It indicated that the reaction of crosslinking by the unsaturated butadiene units occurred again at a higher temperature. ${ }^{29}$ In the meantime, the molecular weight of sols decreased and the PDI increased as the reaction temperature rose (Table 4), revealing that some main chains were broken at the higher temperature.

In the previous study, ${ }^{19}$ we found that devulcanizing reagent was necessary for the devulcanizing reaction in $\mathrm{ScCO}_{2}$. In order to analyze the influence of temperature with devulcanizing reagent in supercritical ethanol, DPDSs in various concentrations were added in the reaction. The result is presented in Figure 5. It can be seen that the SF increased as the temperature rose at a certain

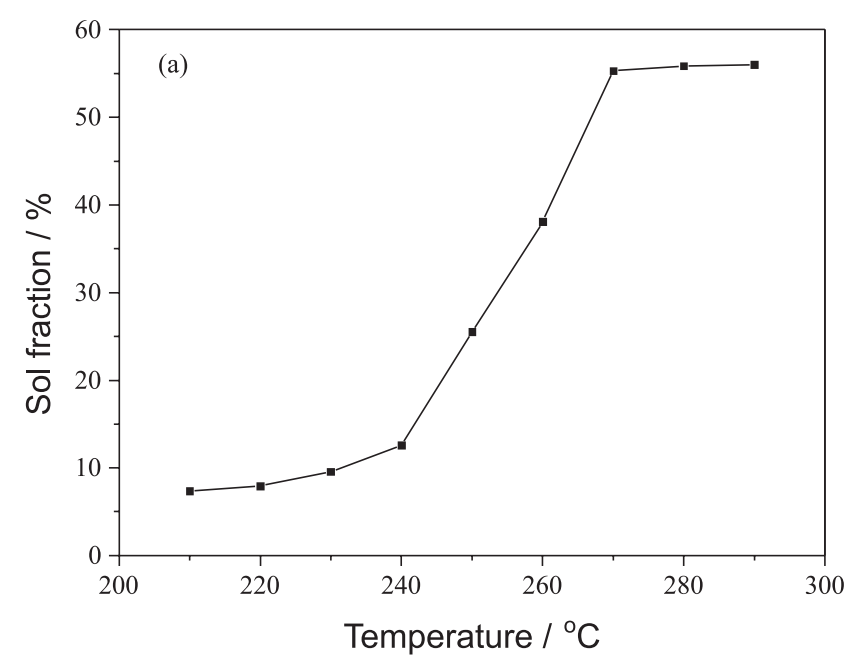

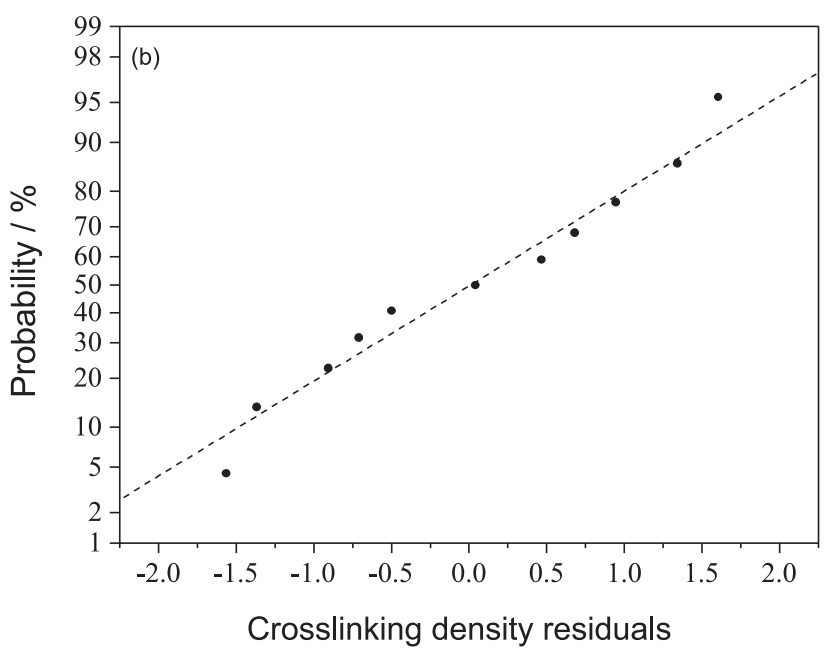

Table 4. Characters of sol at various reaction temperatures

\begin{tabular}{lccc}
\hline Temperature $/{ }^{\circ} \mathrm{C}$ & $\mathrm{Mn}^{\mathrm{a}} /\left(\mathrm{g} \mathrm{mol}^{-1}\right)$ & $\mathrm{Mw}^{\mathrm{b}} /\left(\mathrm{g} \mathrm{mol}^{-1}\right)$ & $\mathrm{PDI}^{\mathrm{c}}$ \\
\hline 230 & 64659 & 88937 & 1.375 \\
250 & 40362 & 67903 & 1.682 \\
270 & 37556 & 74583 & 1.986 \\
\hline
\end{tabular}

${ }^{a}$ Number average molecular weight; ${ }^{b}$ weight average molecular weight; cpolymer dispersity index.

concentration of DPDS. In the case of a relatively low temperature such as $250{ }^{\circ} \mathrm{C}$, the SF increased obviously from 25 to $51 \%$ with an increase of DPDS from 0 to $12 \mathrm{~g} \mathrm{~L}^{-1}$. However, the influence of DPDS was not obvious when the temperature reached a relatively high plateau such as $270^{\circ} \mathrm{C}$. This result demonstrated that the devulcanizing reagent could accelerate the reaction rate at a relatively low temperature, but the effect was waning with the increase in temperature.

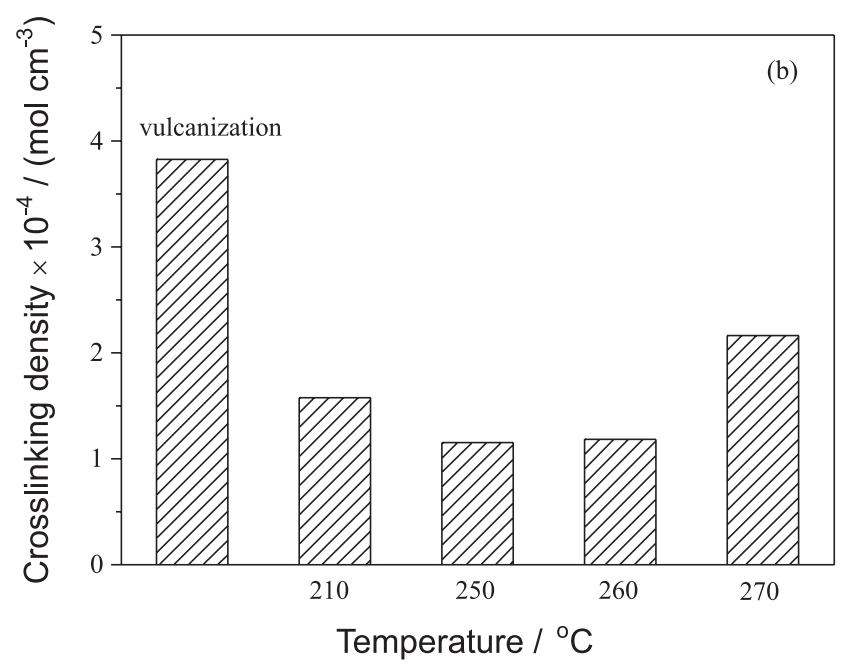

Figure 4. Effects of reactive temperature on (a) sols and (b) $\mathrm{CD}$ of gels. Reaction conditions: 8 MPa pressure, 60 min time. 


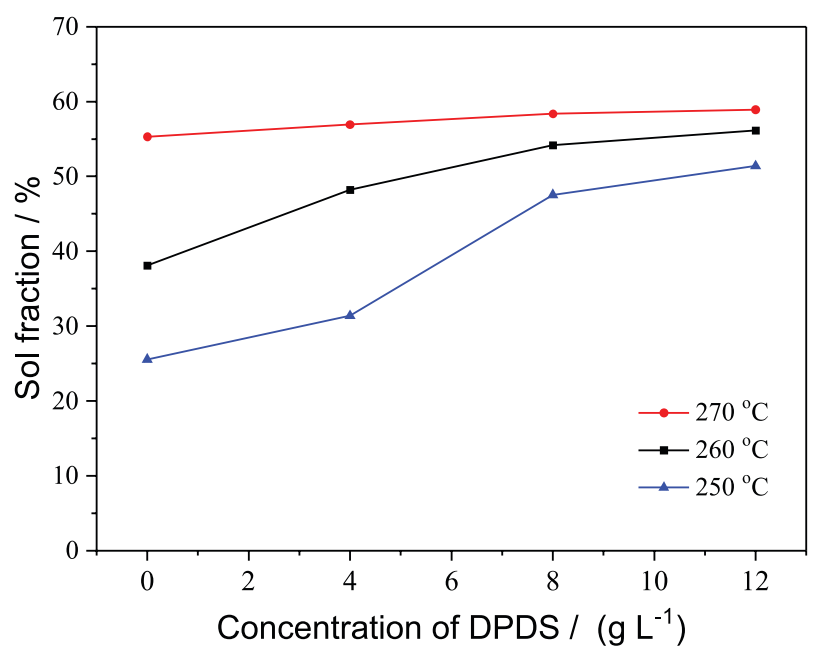

Figure 5. Effect of DPDS on devulcanization in supercritical ethanol. Reaction conditions: 8 MPa pressure, 60 min time.

In order to explore the effect of temperature on devulcanization mechanism in supercritical ethanol, the devulcanized samples compared with the vulcanizate were characterized and analyzed. All the devulcanized samples were obtained under the conditions of $8 \mathrm{MPa}$ and $60 \mathrm{~min}$.

\section{Thermal analysis}

The results of thermal analysis of vulcanizate and devulcanized gels are shown in Figure 6. The TG curve shapes between 300 and $500{ }^{\circ} \mathrm{C}$ are almost the same (Figure 6a), indicating that the thermostability of devulcanized gels was similar to the vulcanizate. Moreover, the weight loss of devulcanized gels from 300 to $500{ }^{\circ} \mathrm{C}$ decreased gradually with an increase in the reaction temperature. This can be attributed to the decrease of the polymer fraction in gels, which was in good conformity with the result in Figure 4a. Notably, the weight loss curve

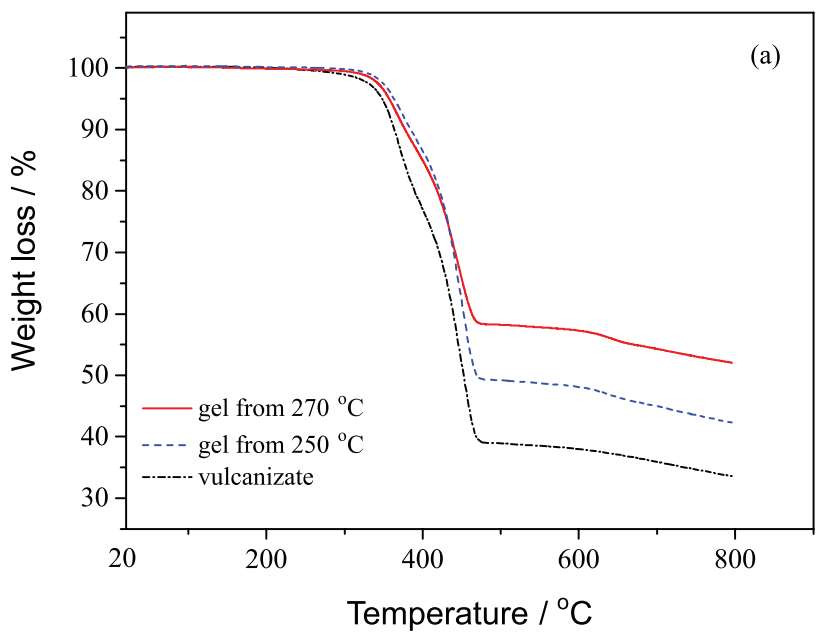

of devulcanized gel shows a deviation to higher temperature owing to the proportional change of NR and BR in gels. ${ }^{30}$ In DTG, the peak intensity at $375^{\circ} \mathrm{C}$ is weakened gradually with an increase in temperature (Figure 6b). Through fitting analysis, the proportion of NR and BR in gels is shown in Table 5. It can be found that, after the devulcanization at 250 and $270{ }^{\circ} \mathrm{C}$, the percentages of NR $(39.2$ and $33.5 \%$, respectively) in gels reduced significantly in comparision with that in vulcanizate $(53.6 \%)$. Correspondingly, the ratios of NR/BR among the three samples, vulcanizate and devulcanized gels at 250 and $270{ }^{\circ} \mathrm{C}$, tended to decrease. These results implied that the devulcanizing reaction rate of NR was greater than that of BR in supercritical ethanol.

Table 5. Mass percentage of NR and BR through the DTG analysis

\begin{tabular}{lccccc}
\hline \multirow{2}{*}{ Sample } & \multicolumn{2}{c}{ Peak position $/{ }^{\circ} \mathrm{C}$} & \multicolumn{2}{c}{ Proportion $/ \%$} & \multirow{2}{*}{$\begin{array}{c}\text { NR/BR } \\
\text { ratio }\end{array}$} \\
\cline { 2 - 5 } & $\mathrm{NR}$ & $\mathrm{BR}$ & $\mathrm{NR}$ & $\mathrm{BR}$ & 4.15 \\
\hline Vulcanizate & 375 & 455 & 53.6 & 46.4 & 1.15 \\
Gel at $250{ }^{\circ} \mathrm{C}$ & 375 & 455 & 39.2 & 60.8 & 0.64 \\
Gel at $270{ }^{\circ} \mathrm{C}$ & 375 & 455 & 33.5 & 66.5 & 0.50 \\
\hline
\end{tabular}

NR: natural rubber; BR: butadiene rubber.

Results of DSC analysis of vulcanizate and devulcanized gels are shown in Figure 7. Clearly, there were two glass transitions in the DSC curve of vulcanizate (in Figure 7a). However, the glass transition at $-65{ }^{\circ} \mathrm{C}$, according to NR, was not obvious in devulcanized gels (Figures $7 \mathrm{~b}$ and 7c). This change implied that the most NR had been devulcanized and formed the sol in samples $\mathrm{b}$ and $\mathrm{c}$, whose only glass transitions at -94 and $-74{ }^{\circ} \mathrm{C}$, respectively, were due to BR. In general, the mobility of polymer chains was strengthened with a decrease in $\mathrm{CD}$, which would result in a lower glass transition temperature. However, Levin et al..$^{31}$ found that the mono-, di- and polysulfur

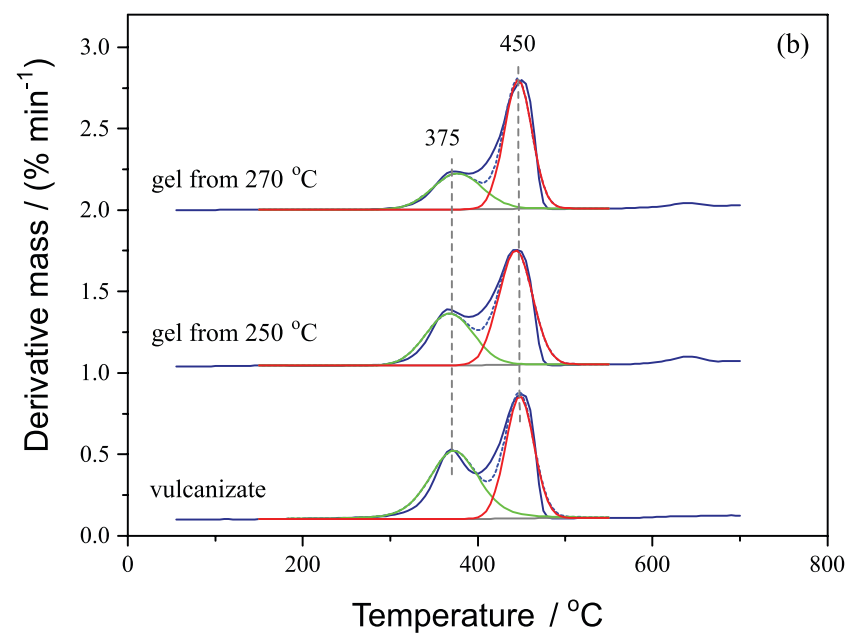

Figure 6. Thermal analysis of vulcanizate and devulcanized gels. (a) TG and (b) DTG. 
bonds would rearrange into cyclic sulfur structures at a higher temperature, which might decrease the molecular mobility in polymer and result in a significant increase of glass transition temperature. Based on this explanation, the significantly increase in the glass transition temperature of gels (b and c) whose main polymer component was BR should be due to the rearrangement of bonds.

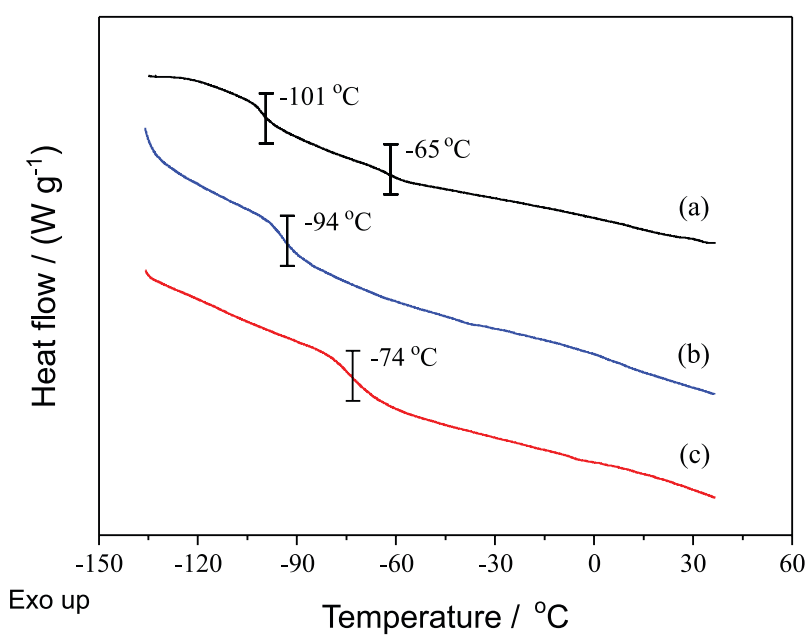

Figure 7. DSC analysis of vulcanizate and devulcanized gels. (a) Vulcanizate; (b) gel from devulcanization at $250^{\circ} \mathrm{C}$ and (c) gel from devulcanization at $270{ }^{\circ} \mathrm{C}$.

\section{Structure analysis of sols at various reaction temperatures}

The FTIR analysis of sols at different reaction temperatures is shown in Figure 8. The characteristic bands around 500,570 and $730 \mathrm{~cm}^{-1}$ refer to the presence of S-S and C-S groups. ${ }^{32,33}$ It can be observed that the band of S-S $\left(500 \mathrm{~cm}^{-1}\right)$ almost disappeared at different temperatures, confirming the breaking of a large proportion of the crosslink bonds, i.e., the success of the devulcanization treatment. ${ }^{34}$

The bands located at 835 and $1372 \mathrm{~cm}^{-1}$, attributed to $\mathrm{C}=\mathrm{CH}$ stretching vibration and the $-\mathrm{CH}_{3}$ stretching vibration, respectively, confirm the presence of NR in the sol. ${ }^{35,36}$ The intensity of these two bands does not appreciably change with increment of treatment temperature, meaning that the $\mathrm{C}=\mathrm{C}$ double bonds were not destroyed notably by the temperature rise. Therefore, a relatively complete polymer structure was maintained in sol.

The characteristic band at $970 \mathrm{~cm}^{-1}$ (the out-of-plane bending vibrations of $\mathrm{C}-\mathrm{H}$ of trans $-\mathrm{CH}=\mathrm{CH}-$ ) corresponds to butadiene in BR. ${ }^{26,30,37}$ It is worth noting that, compared with other characteristic bands, the intensity of this band decreased with an increase in the reaction temperature, implying that the proportion of BR in the sol was reduced. It also proved that more NR was devulcanized to form the sol at higher temperature. This result agrees well with the change of NR/BR ratio in Table 5.

The bands between 1800 and $1500 \mathrm{~cm}^{-1}$, corresponding to the $\mathrm{C}=\mathrm{C}$ and $\mathrm{C}=\mathrm{O}$ bonds, show that few changes take place at different temperatures. ${ }^{8,34}$ This result is in agreement with the analysis above, indicating that the supercritical ethanol could make the main chain structure maintain integrality.

Band of $\mathrm{C}-\mathrm{H}$ bond located in the range from 3100 to $2750 \mathrm{~cm}^{-1}$ does not show differences at different temperatures, ${ }^{38,39}$ suggesting that the hydrocarbon backbone of sidewall rubber was not destroyed by higher temperature in supercritical ethanol.

In the general process of devulcanization reaction, there is an inverse relationship between the SF and CD of gel. The $\mathrm{CD}$ of the vulcanized rubber gradually declines until the polymer chain breaks away from the crosslinking network, and a sol is formed. However, the SF and CD of gel, here, increase simultaneously with the rising of temperature as shown in Figure 4. According to the analysis of DTG and FTIR, the main composition of sols is NR. Correspondingly, the main composition of gels is BR. The

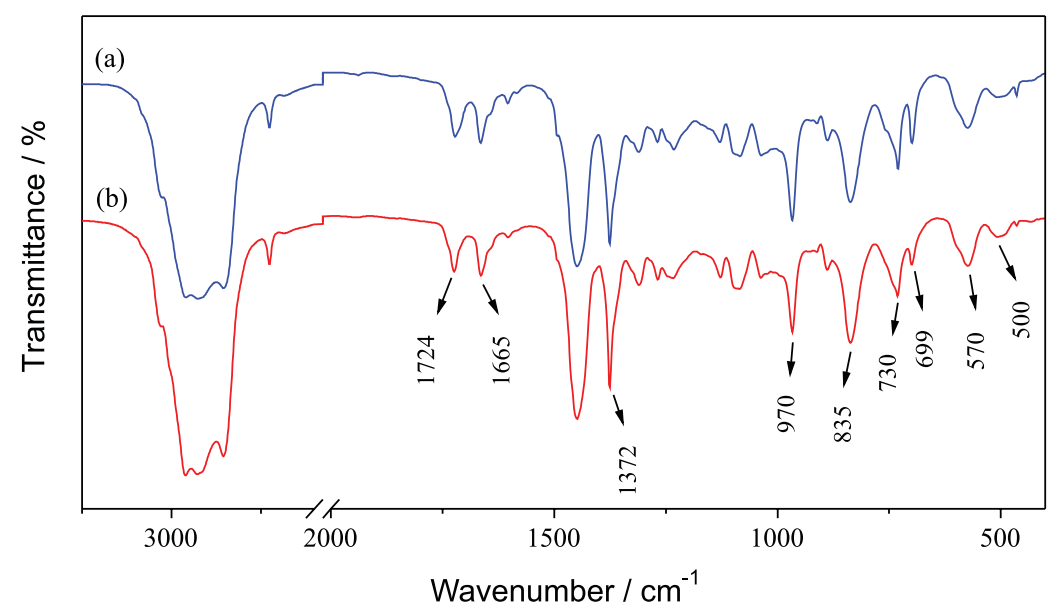

Figure 8. FTIR of sols at different reaction temperatures. (a) Sol from devulcanization at $250{ }^{\circ} \mathrm{C}$ and (b) sol from devulcanization at $270{ }^{\circ} \mathrm{C}$. 
high temperature causes more devulcanization of NR, but the increase in the $\mathrm{CD}$ of $\mathrm{BR}$.

\section{Model analysis of crosslinked network scission}

Horikx's theory ${ }^{40}$ was used to determine the breakage mechanism of crosslinked network during devulcanization. Figure 9 presents SF from devulcanizing sidewall rubber as a function of the relative decrease in $\mathrm{CD}$ at different temperatures. The solid curve corresponds to the devulcanizing process in which only crosslink bonds scission took place, and the dashed curve corresponds to the devulcanizing process in which only main chains scission occurred. When the temperature was $210^{\circ} \mathrm{C}$, the analysis result fell near the solid curve, indicating that the formation of sol was mainly caused by crosslink bonds scission. The result was close to the dashed curve as the temperature reached $250{ }^{\circ} \mathrm{C}$, implying that the breakage of main chain gradually increased with the rising of temperature. When the temperature reached 260 or $270{ }^{\circ} \mathrm{C}$, the results all fell above the dashed curve, indicating that the devulcanization was mainly caused by main chains scission. Obviously, main chains scission gradually predominated with the rise of temperature during devulcanization. It means that the increase of SF was mainly dependent on the breakage of main chains.

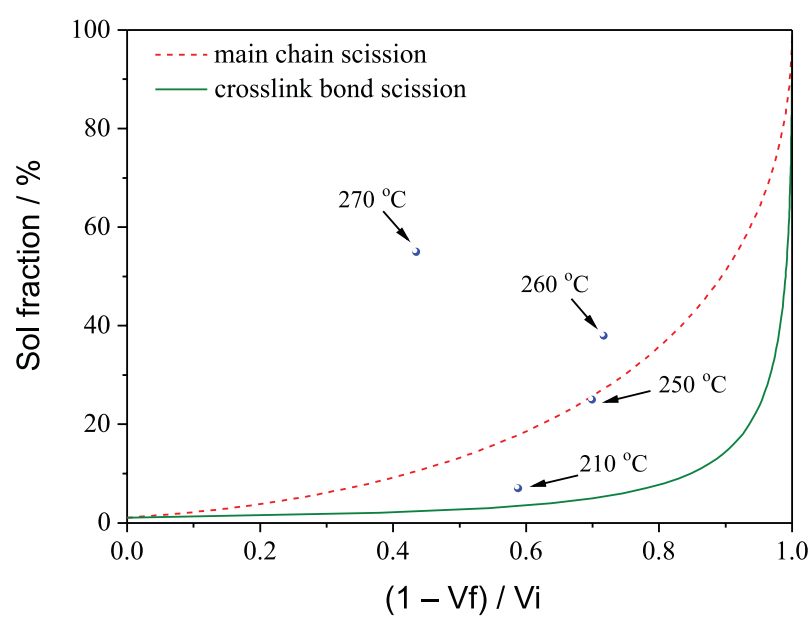

Figure 9. SF of devulcanized sidewall rubber against relative decrease in $\mathrm{CD}$ at different reaction temperatures.

\section{Devulcanizing reaction rate}

The reaction kinetics was used to further analyze the supercritical ethanol devulcanizing process. The objective function was gel concentration, and the calculation method is shown as equation 4 . The initial gel concentration was $100 \%$.

Gel concentration $\left(\mathrm{mg} \mathrm{cm}^{-3}\right)=\frac{\mathrm{W}_{\mathrm{d}} \times \mathrm{w}-\mathrm{W}_{\mathrm{s}}}{\text { Reactor volume }\left(\mathrm{cm}^{3}\right)}$
It was assumed that the devulcanizing process followed the first-order kinetics. ${ }^{14,20}$ The experimental results are shown in Figure 10. The first-order kinetics equation is shown as

$-\frac{\mathrm{dC}_{\mathrm{gel}}}{\mathrm{dt}}=\mathrm{kC}_{\mathrm{gel}}$

where $\mathrm{C}_{\text {gel }}$ is the gel concentration, $\mathrm{t}$ is the reaction time and $\mathrm{k}$ is the reaction rate constant.

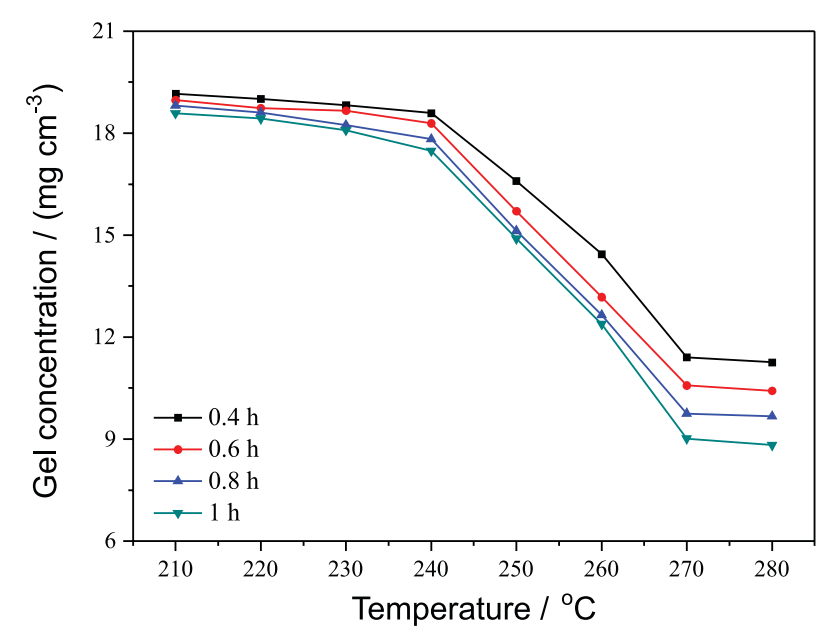

Figure 10. Experimental results for reaction kinetics.

The following equations 6 and 7 can be obtained by integrating equation 5 from $t_{0}$ to $t$ (starting time and reaction time, respectively). In this study, the reaction time during the elevation of temperature was ignored, and the integral was performed only within the isothermal period, thus the starting time $\mathrm{t}_{0}$ was 0 .

$$
\begin{aligned}
& -\int_{\mathrm{C}_{0, \mathrm{gel}}}^{\mathrm{C}_{\mathrm{gel}}} \frac{\mathrm{dC}_{\mathrm{gel}}}{\mathrm{C}_{\mathrm{gel}}}=\mathrm{k} \int_{\mathrm{t}_{0}}^{\mathrm{t}} \mathrm{dt} \\
& -\ln \mathrm{C}_{\mathrm{gel}}=\mathrm{kt}-\ln \mathrm{C}_{\mathrm{gel}, 0}
\end{aligned}
$$

where $\mathrm{C}_{\mathrm{gel}, 0}$ is gel concentration at $\mathrm{t}=\mathrm{t}_{0}$. The results of plots in Figure 11a were obtained by equation 7. Further, the lines were calculated by the least-squared method and fitted well the measured variation of the gel concentration. The $\mathrm{k}$ value was calculated by the slope of lines as presented in Figure 11b. It can be observed that the k rose rapidly as the temperature was above $240{ }^{\circ} \mathrm{C}$. This result further demonstrated that supercritical ethanol played an important role in promoting devulcanization.

\section{Discussion}

In general, the crosslinked network is formed by mono-, 

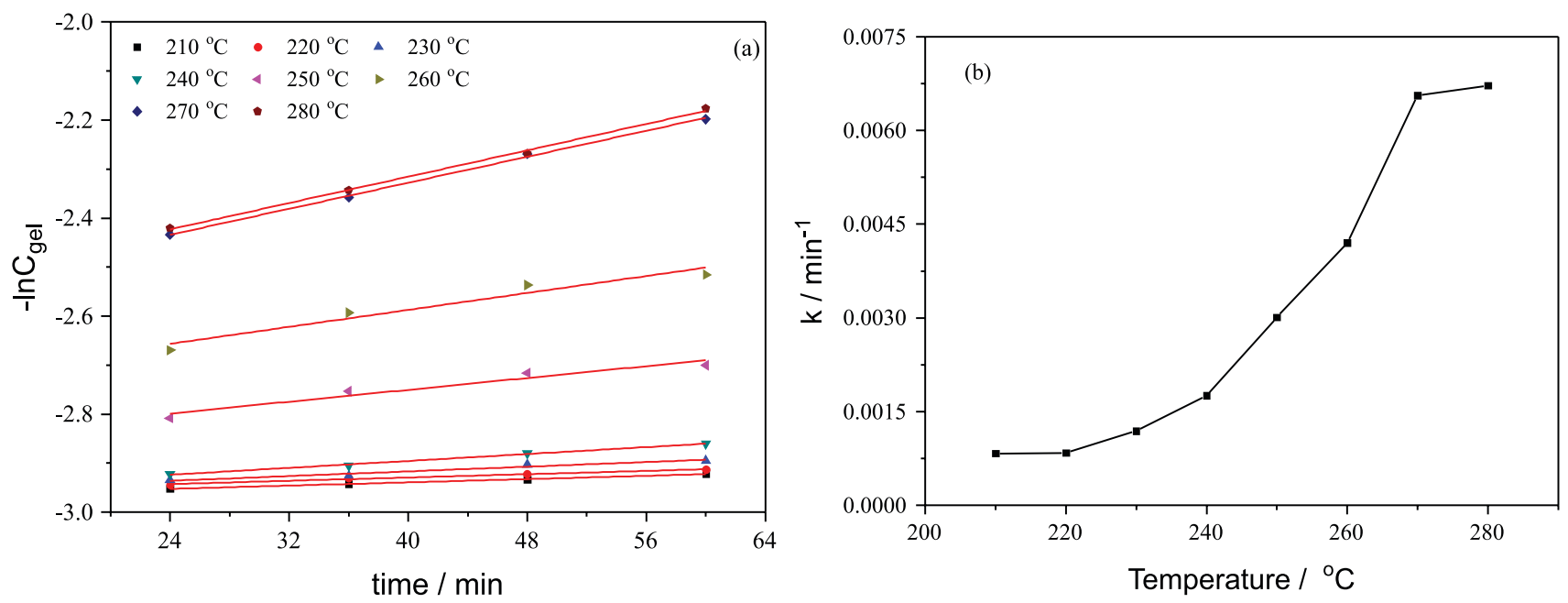

Figure 11. Reaction rate constant. (a) $-\operatorname{lnC}_{\text {gel }} v s$. time and (b) $\mathrm{k}$ value $v s$. temperature.

di- and polysulfur bonds. ${ }^{41,42}$ In this study, with the increase in temperature, not only the crosslink bonds but also some main chains were broken in supercritical ethanol process (Figure 9), which all had great influence on SF.

In Figure 4, there can be three regions of temperature: a low temperature region below $240{ }^{\circ} \mathrm{C}$ in subcritical state, a medium temperature region from 240 to $270{ }^{\circ} \mathrm{C}$ and a high temperature region above $270{ }^{\circ} \mathrm{C}$. In the low temperature region, the breakage of crosslinked bonds predominated the devulcanizing process, but reaction rate was relatively slow.

As the temperature reached the medium temperature region, the ethanol transferred to supercritical state. On one hand, the number of radicals increased due to the promotion on dynamics. The radicals had an instinctive tendency to couple and then form macromolecular structure fragments, which was the main compositions of sols. ${ }^{43} \mathrm{On}$ the other hand, the reaction activity of supercritical ethanol enhanced. As a result, the crosslinked structure broke rapidly and the sols increased sharply, with the unexpected occurrence of the main chain scission. Interestingly, due to the different structure properties between NR and BR, NR was easier to be devulcanized than BR as can be seen from the results of DTG and FTIR.

When the temperature further rose above $270^{\circ} \mathrm{C}$ into the high temperature region, the degradation rate of crosslinked structure was fast enough to ignore the effect of DPDS. Meanwhile, the main chains continued breaking, which predominated in the devulcanizing process. In addition, the cyclic sulfide structure transformed from the rearrangement of crosslinking bonds grafted onto the main chains, which resulted in the increase of glass transition temperature (as shown in Figure 7).

Based on the presented discussion, the proposed mechanisms for both NR and BR are presented in
Schemes 1 and 2. According to the result of Figure 4b, the unsaturated butadiene units in BR would transform to the crosslink structure at a high temperature as a result of devulcanization by ethanol. The possible reaction process of increase in CD is also proposed in Scheme 3.

\section{Conclusions}

The effect of temperature on devulcanization for sidewall rubber in supercritical ethanol was studied. A full factorial experimental design was used to define the experimental conditions. Reaction temperature was the most important factor in the devulcanization process. Reaction pressure above critical value and reaction time exert no significant influence. When the reaction temperature changed from 240 to $270{ }^{\circ} \mathrm{C}$, the SF rose dramatically from 13 to $55 \%$. Meanwhile, the CD of devulcanized gel drastically reduced comparing with that of vulcanizate. The use of devulcanizing reagent did not significantly affect the SF at a high temperature of $270{ }^{\circ} \mathrm{C}$.

Devulcanizing reaction rate of NR was faster than that of BR in supercritical ethanol. The mass percentage of NR/ $\mathrm{BR}$ in gels continued to decrease with the rise in reaction temperature as revealed by DTG analysis, which was in agreement with the results of DSC and FTIR.

The scission of the crosslink bonds predominated in devulcanization process at lower temperatures and the main chains began to be broken when the temperature reached $250{ }^{\circ} \mathrm{C}$, which has been proved by the mode analysis of the crosslinked network scission. The glass transition temperature of the gels increased with the rise in reaction temperature because of the reduction in the mobility of main chains, which was caused by the cyclic sulfur structure. The kinetic constant $\mathrm{k}$ rose significantly 
<smiles>CCC=C(C)CC1C=C(C)CCC(C=C(C)CC)SS1</smiles>

(1)<smiles>CCC=CCC(S)C=C(C)CC</smiles><smiles>CCC=C(C)CC(N)C=C(C)CC</smiles>

\section{(2)}<smiles>CCC=C(C)CC(S)C=C(C)CC</smiles><smiles>CCC=C(C)CC1CSCCC1(C)CC</smiles><smiles>CCC=C(C)CC1CC(C)=CCCCC1C=C(C)CC</smiles>

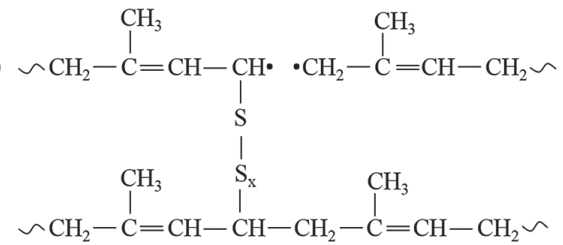

(3)

(a) (b) (c) (d) $\underset{\text { Coupling }}{\longrightarrow}$ Sol component

Scheme 1. Simplified mechanism proposed for the formation of sol during devulcanization of NR by supercritical ethanol.

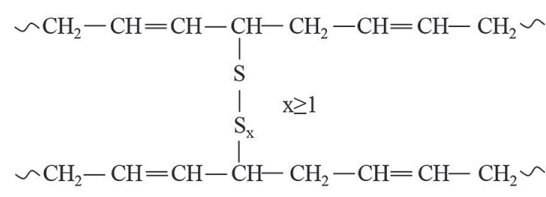

\section{(1)}

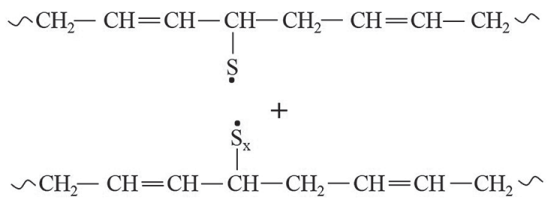

(2)

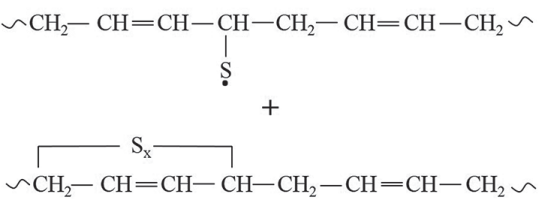

(c)
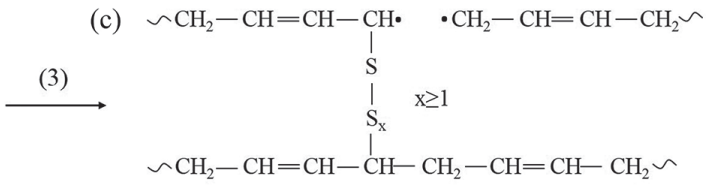

(a)

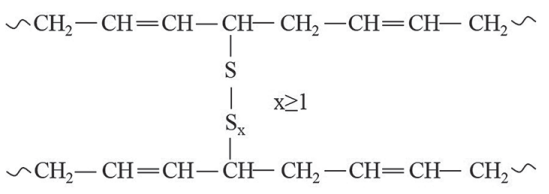

(a) (b) (c) (d) $\underset{\text { Coupling }}{\longrightarrow}$ Sol component

Scheme 2. Simplified mechanism proposed for the formation of sol during devulcanization of BR by supercritical ethanol.

when the temperature was above $240{ }^{\circ} \mathrm{C}$, which indicated that temperature had the most notable promoting effect in the supercritical ethanol devulcanizing process.

\section{Acknowledgments}

This work is supported by National Natural Science Foundation of China (51703013). 


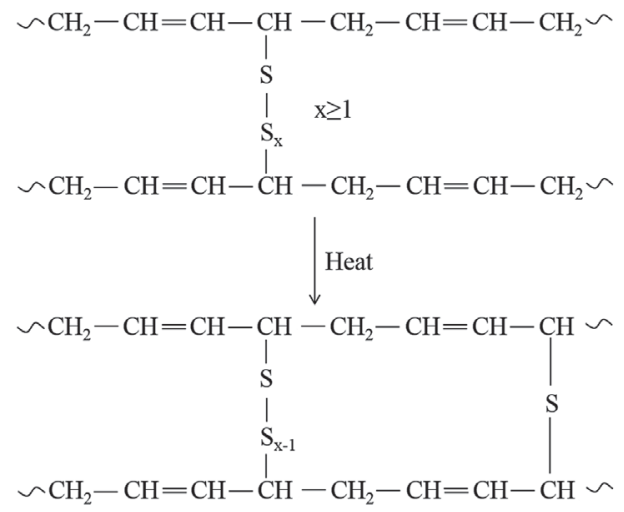

Scheme 3. The crosslinking reaction caused by unsaturated butadiene units at high temperature.

\section{References}

1. Srinivasan, A.; Sanmugharaj, A. M.; Bhowmick, A. K. In Current Topics in Elastomer Research; Bhowmick, A. K., ed.; CRC Press: Boca Raton, 2008.

2. Rajan, V. V.; Dierkes, W. K.; Joseph, R.; Noordermeer, J. W. M.; Prog. Polym. Sci. 2006, 31, 811.

3. Verbruggen, M. A. L.; Van der Does, L.; Noordermeer, J. W. M.; Van Duin, M.; J. Appl. Polym. Sci. 2008, 109, 976.

4. Zhang, X. X.; Lu, Z. X.; Dong, T.; Li, H.; Lu, C. H.; J. Appl. Polym. Sci. 2013, 127, 4006.

5. Maridass, B.; Gupta, B. R.; Polym. Test. 2004, 23, 377.

6. Ghosh, J.; Ghorai, S.; Bhunia, S.; Roy, M.; De, D.; Polym. Eng. Sci. 2018, 58, 74 .

7. de Sousa, F. D. B.; Scuracchio, C. H.; Hu, G. H.; Hoppe, S.; J. Appl. Polym. Sci. 2016, 133, ID 43503. DOI 10.1002/ APP.43503.

8. de Sousa, F. D. B.; Scuracchio, C. H.; Hu, G. H.; Hoppe, S.; Polym. Degrad. Stab. 2017, 138, 169.

9. Zanchet, A.; Carli, L. N.; Giovanela, M.; Brandalise, R. N.; Crespo, J. S.; Mater. Des. 2012, 39, 437.

10. Isayev, A. I.; Rubber Chem. Technol. 1995, 68, 267.

11. Mangili, I.; Lasagni, M.; Huang, K.; Isayev, A. I.; Chemom. Intell. Lab. Syst. 2015, 144, 1.

12. Sato, S.; Honda, Y.; Kuwahara, M.; Kishimoto, H.; Yagi, N.; Muraoka, K.; Watanabe, T.; Biomacromolecules 2004, 5, 511.

13. Tsuchii, A.; Tokiwa, Y.; J. Polym. Environ. 2006, 14, 403.

14. Goto, T.; Yamazaki, T.; Sugeta, T.; Okajima, I.; Sako, T.; J. Appl. Polym. Sci. 2010, 109, 144.

15. Cheng, S.; Wilks, C.; Yuan, Z.; Leitch, M.; Xu, C.; Polym. Degrad. Stab. 2012, 97, 839.

16. Kershaw, J. R.; Fuel 1998, 77, 1113.

17. Li, X.; Liu, Z.; Wang, X.; Liu, F.; Wei, W.; Zhang, R.; Li, Z.; Xu, X.; Polym. Mater.: Sci. Eng. 2016, 32, 112.

18. Mangili, I.; Oliveri, M.; Anzano, M.; Collina, E.; Pitea, D.; Lasagni, M.; J. Supercrit. Fluids 2014, 92, 249.
19. Liu, Z.; Li, X.; Xu, X.; Wang, X.; Dong, C.; Liu, F.; Wei, W.; Polym. Degrad. Stab. 2015, 119, 198.

20. Lee, H. S.; Ju, H. J.; Cho, H. K.; Koo, C. M.; Hong, S. M.; Kim, H.; Lee, Y. W.; Polym. Degrad. Stab. 2008, 93, 2084.

21. Su, X.; Zhao, Y.; Zhang, R.; Bi, J.; Fuel Process. Technol. 2004, $85,1249$.

22. Flory, P. J.; Rehner, J.; J. Chem. Phys. 1943, 11, 521.

23. Sheehan, C. J.; Bisio, A. L.; Rubber Chem. Technol. 1966, 39 , 149.

24. Porter, M.; Rubber Chem. Technol. 1967, 40, 866.

25. Fukumori, K.; Matsushita, M.; Okamoto, H.; Sato, N.; Suzuki, Y.; Takeuchi, K.; JSAE Rev. 2002, 23, 259.

26. Ramarad, S.; Khalid, M.; Ratnam, C. T.; Chuah, A. L.; Rashmi, W.; Prog. Mater. Sci. 2015, 72, 100.

27. Naskar, A. K.; De, S. K.; Bhowmick, A. K.; Pramanik, P. K.; Mukhopadhyay, R.; Rubber Chem. Technol. 2000, 73, 902.

28. Klingensmith, B.; Rubber World 1991, 203, 16.

29. Sarkar, M. D.; Mukunda, P. G.; De, P. P.; Bhowmick, A. K.; Rubber Chem. Technol. 1997, 70, 855.

30. Fernández-Berridi, M. J.; González, N.; Mugica, A.; Bernicot, C.; Thermochim. Acta 2006, 444, 65.

31. Levin, V. Y.; Kim, S. H.; Isayev, A. I.; Massey, J.; Meerwall, E. V.; Rubber Chem. Technol. 1996, 69, 104.

32. Tao, G.; He, Q.; Xia, Y.; Jia, G.; Yang, H.; Ma, W.; J. Appl. Polym. Sci. 2013, 129, 2598.

33. Colom, X.; Faliq, A.; Formela, K.; Cañavatea, J.; Polym. Test. 2016, 52, 200.

34. Aoudia, K.; Azem, S.; Ait Hocine, N.; Gratton, M.; Pettarin, V.; Seghar, S.; Waste Manage. 2017, 60, 471.

35. Rooj, S.; Basak, G. C.; Maji, P. K.; Bhowmick, A. K.; J. Polym. Environ. 2011, 19, 382.

36. Jana, G. K.; Das, C. K.; Polym.-Plast. Technol. Eng. 2005, 44, 1399.

37. Masson, J.; Pelletier, L.; Collins, P.; J. Appl. Polym. Sci. 2015, 79, 1034.

38. Karabork, F.; Pehlivan, E.; Akdemir, A.; J. Polym. Eng. 2014, $34,543$.

39. Li, Y.; Zhao, S.; Wang, Y.; J. Polym. Environ. 2012, 20, 372.

40. Horikx, M. M.; J. Polym. Sci. 1956, 29, 1166.

41. Holst, O.; Stenberg, B.; Christiansson, M.; Biodegradation 1998, 9, 301.

42. Kojima, M.; Ogawa, K.; Mizoshima, H.; Tosaka, M.; Kohjiya, S.; Ikeda, Y.; Rubber Chem. Technol. 2003, 76, 957.

43. Jiang, K.; Shi, J.; Ge, Y.; Rui, Z.; Yao, P.; Li, X.; Zhang, L.; J. Appl. Polym. Sci. 2013, 127, 2397.

Submitted: February 12, 2018 Published online: May 14, 2018 